\title{
2D mammography and breast cancer screening: a global and local perspective
}

\author{
U.A. Liyanage \\ Faculty of Medicine, University of Colombo, Sri Lanka
}

Key words: Breast screening; digital mammography; Sri Lanka

\section{Introduction}

Radiological imaging plays a pivotal role in early detection of breast cancer. The basic two view mammography (craniocaudal and mediolateral oblique views - CC and MLO) has been the traditional tool in screening for breast cancer. Nevertheless, an overall 15 $30 \%$ of breast cancer is not diagnosed by routine mammographic screening [1]. In the subgroup of women with dense breasts, this percentage is even higher [1, 2, 3, 4]. Hence, several new advances have emerged in the field of breast imaging with prospects for improving early detection of breast cancer.

Conventional analogue mammography (film) is being replaced by high technology digital mammography (digital) worldwide. The trend to invest in digital mode is now seen in lower middle-income countries like Sri Lanka as well.

The digital mode has further evolved to introduce new advances to breast imaging such as digital breast tomosynthesis (DBT) which allows the breast to be imaged in thin cross sections, reducing the burden of tissue overlap. 2D synthetic mammography is another high-tech development in DBT promising to reduce the burden of radiation in DBT and 2D combined protocols.

Parallel to the technological advances in mammography, the guidelines for mammographic breast screening has evolved in keeping with evidence mostly from nationwide breast screening programmes. Professional bodies, authorities and consensus groups of different countries have tabled age and interval recommendations for mammographic breast screening catering to target populations of their countries.

Correspondence: U.A. Liyanage

E-mail: udari8@gmail.com
Although the breast is amenable to imaging by a number of imaging modalities ranging from conventional 2D mammography and ultrasound scan (USS) to tomosynthesis and magnetic resonance imaging (MRI), not all imaging modalities are recognized as routine screening tools for breast cancer. The role of digital breast tomosynthesis, 2D synthetic mammography and adjunct breast imaging modalities such as USS and MRI in breast cancer screening will be discussed in a subsequent paper.

\section{Mammography}

Mammography is indicated both for screening of breast cancer and for further assessment of otherwise detected breast pathology.

In countries with well-established breast screening programmes, women are individually invited for routine screening at regular intervals and are methodically followed up either by routine recall or by recall for further assessment of screening-detected significant abnormalities. Assessment may involve further mammographic views, breast USS and image-guided tissue sampling followed by referral for further care and follow up. Such systematic screening programs include professional and methodical delivery of results to the identified parties as well as organised data collection, regular monitoring and evaluation of the screening programme, and an authorised body responsible for delivery of care, maintenance and upgrading of service quality [5].

\section{Benefits of mammographic breast screening}

Mammographic breast screening has been historically valued as a versatile programme to reduce mortality from breast cancer due to its ability for early detection of malignancy when cancer is still at a curable stage [6]. Benefits of mammographic breast screening vary for different age groups. 
The benefits of a screening mammography for the 50 to 59 year age group has been most clearly stated in terms of relative reduction of mortality from breast cancer. Figures for overall mortality reduction are $15-20 \%$ for this age group [7, 8]. Furthermore, the International Agency for Research on Cancer (IARC) workgroup on breast cancer (2014) reports even higher figures for reduction in risk of mortality due to breast cancer for the 50-59 year age group who actually participated in the screening programme. Their published figures reach $40 \%$ for those women who actually participated in a screening programme as compared to $23 \%$ observed in women who were invited for screening in the same age group [5]. Mammographic screening is reported to reduce mortality from breast cancer in the 60 to 69 year age group as well $[5,7,9]$. There was debate on the efficacy of mortality reduction for the 70 to 74 year age group, but there is sufficient evidence that the screening mammography reduces mortality from breast cancer in this age group as well $[5,10]$.

Unlike for the 50 to 74 year age group, there is much debate on the adequacy of global evidence for younger women, particularly of the 40-49 year age group. Mortality reduction data for the 39 to 49 year age group was revised in a meta-analysis of 8 trials by Nelson HD et al for a report on The Systematic Evidence Review Update for the U.S. Preventive Services Task Force (USPSTF) - 2009. It reported benefits similar to the 50 59 year age group (relative risk [RR] $0.85 ; 95 \%$ credible interval [CrI], 0.75-0.96; 8 trials) [7, 9]. However, The IARC workgroup on breast cancer (2014) concludes that there is only limited evidence for significant reduction of mortality from breast cancer detected by mammographic screening for the 40 - 44 year age group. Similarly, there is limited evidence of mortality reduction for the 45 to 49 year age group as well. Nevertheless, it is interesting to note that the IARC report notes that even though the majority vote was for limited evidence, there was almost equal voting amongst voting members for sufficient evidence in mortality reduction for the 45 - 49 year age group [5].

However, even though there is sufficient evidence that the benefits of mammographic breast screening substantially outweighs the risk of incurring radiationinduced breast cancer due to mammographic breast screening in the 50 - 74 year age group of the population, similar evidence is not clear for the younger 40 - 49 year age group [5].

Disadvantages of screening mammography - over diagnosis

In addition to the widely accepted advantages, mammographic breast screening has recognised disadvantages such as over diagnosis, false positive outcomes and radiation caused breast malignancy [5]. The issue of over diagnosis has recently become a widely discussed topic. Mammography detects occult malignancies which may never have surfaced in a woman's life time if not for screening. In the absence of clear markers to identify life-threatening screeningdetected malignancies from cancer that would not surface clinically, or cancer which would not become life-threatening during a woman's life time, all cancers are treated and therefore the latter group of women will experience only the risks and side effects of treatment [8]. Hence, over diagnosis creates great physical and psychological burdens on the affected women as well as an additional cost to the financial burden of population screening. These disadvantages need to be weighed against mortality reduction and the other benefits of mammographic screening.

The view point of the IARC working group on breast cancer screening (2014) is that currently there is sufficient evidence to conclude that mammographic screening leads to over diagnoisis of breast cancer [5]. However, in a Swedish randomized trial report, Duffy et al found that the benefits of mammographic screening in terms of lives saved largely outweighs the harm that may occur due to over diagnosis. The trial further concludes that there are an additional $2-2.5$ lives saved for every over diagnosed case [11].

Target age group for screening and routine screening interval

In addition to evidence on mortality reduction, population specific data on the age group related incidence of breast cancer, peak incidence age group, financial benefit from life years saved and other public health directives of a given country are considered when age and interval recommendations are decided in a screening programme.

The target age groups for mammographic breast screening and the recommended routine screening interval varies amongst different countries [Table 1]. 


\begin{tabular}{|c|c|c|}
\hline Country & $\begin{array}{l}\text { Recommended age group for invitation to } \\
\text { the screening programme }\end{array}$ & Recommended screening interval \\
\hline $\mathrm{UK}^{1}$ & $47-50$ to $70-73$ years & 3 yearly \\
\hline $\mathrm{USA}^{2}$ & 40 years onwards ${ }^{3}$ & annually \\
\hline Australia $^{4}$ & 50 to 74 years & 2 yearly \\
\hline
\end{tabular}

Table 1. Recommended guidelines for breast screening in three different countries published by different authorities and consensus groups

1. NHS Breast Cancer Screening Programme - 2015

2. American Cancer Society guidelines for early detection of cancer -2015

3. These guidelines have been challenged by USPSTF report - 2009

4. Breast Screen Australia - 2015

In USA, the majority of professional bodies and consensus groups including the American Cancer Society, the National Cancer Institute, the American College of Radiology and the American College of Surgeons, recommend 40 years as the recruitment age for a screening mammography with an interval of 1 - 2 years [12]. These wide recommendations are challenged by the 2009 USPSTF report which is authored by a group of healthcare professionals making recommendations for preventative care after reviewing the current scientific literature [7, 9]. They recommend 50 years as the starting age for screening with 2 years as the screening interval [13]. Their recommendations against regularly inviting women for screening mammography before the age of 50 has sparked an ongoing debate about breast screening practices and guidelines in the US $[12,14]$. The IARC work group reports that currently there is insufficient data to confirm a most appropriate screening interval [5].

\section{Advances in 2D mammography - digital mode}

Traditionally mammographic screening was carried on a screen film mode (film). With the introduction of digital mammography (digital), the global trend has been to convert breast imaging units to a digital mode.

\section{Is digital mammography better than film mammography?}

Nelson et al (2009) indicated in their report that as the available population studies are based on film mammography there is no published work using digital mammography to assess the efficacy of digital mammographic breast screening. They raised concern on converting to an expensive digital mode purely on an assumption that newer technology is better without valid scientific evidence of its efficacy. They also suggested the possibility of increased false positive results with the digital mode [7,9].

Nevertheless, the screening and diagnostic trial to compare the effectiveness of two types of mammography in detecting breast cancer in women, a multicentre study conducted by Pisano et al (2001) in Canada and USA, has a number of publications on the performance and efficacy of the digital mode. Their Digital Mammographic Imaging Screening Trial (DMIST) group studied the diagnostic accuracy of digital mammography in screening of breast cancer in comparison to film mammography in 49528 women. They concluded that both modalities are of equal efficacy for the entire population, but digital mammography is significantly more accurate for women under 50 years, for women with mammographically dense breasts, and for peri- and postmenopausal women [15].

It was later concluded that the claimed better sensitivity of the digital mode in DMIST actually applies only for the limited subgroup of pre or peri-menopausal women under 50 years of age who have mammographically dense breasts. For all other sub groups, there was no statistically significant difference between the diagnostic accuracy of digital and film mammography. In fact, film mammography has better, but statistically non-significant sensitivity in women with fatty breasts aged 65 years or more [16].

The cost of digital mammography needs to be borne by stakeholders, which could be a 'free' national health system, personal cost to the patient or health insurance. The cost effectiveness of digital mammography was analysed by Tosteson A et al based on the findings of DMIST. They concluded that targeted digital imaging is 
cost effective, whilst offering film to all or offering digital to all women in the screening population is not. Targeted digital imaging is referred to age-targeted digital imaging where this form of imaging is offered to women $<50$ years of age. Tosteson et al also highlighted that the shift from conventional film to the more expensive digital imaging can be considered as beneficial for young ( $<50$ year old) females at the expense of older ( $>65$ year old) women with fatty breasts who may actually benefit more from film mammography. They recommend offering age targeted digital imaging as the most efficient approach for breast screening in USA $[17,18]$.

\section{Mammographic Breast Screening in Sri Lanka}

The National Health System of Sri Lanka and the private sector currently offers opportunistic mammographic breast screening for women on request. The National Cancer Control Programme recommends a screening mammography to be offered for the $50-69$ year age group with a routine screening interval of 2 years [19].

\section{Availability of equipment}

Both the national health sector and the private sector cater for breast imaging in Sri Lanka. Only film mammography was available in both sectors until recently, but the trend to convert to digital mode has been observed in Sri Lanka both in the government and private sector. Digital mammography is now available in the national health sector as well, starting at the National Hospital of Sri Lanka. DBT is currently only available in the private sector. Synthetic mammography facilities are not available in either sector.

The distribution of the imaging units is not uniform and more than $50 \%$ of mammography machines are clustered in the western province in and around Colombo [19]. Introducing mobile screening units which can reach targets in the periphery on a preselected annual date may provide wider coverage for screening in Sri Lanka. Such mobile breast screening units are incorporated in to screening programmes both in developed and developing countries such as India.

\section{Evidence based practice}

The unavailability of a nation-wide mammographic breast screening programme with monitoring and evaluation of screening practices, and the lack of awareness/agreement of current guidelines may lead to varying opportunistic screening practices both in the national health sector and in the private sector.

Considering the limited local resources, newer technology can be offered selectively on an individual basis with the presence of medical evidence. Offering digital screening mammography to the subgroup of women who are $<50$ years, premenopausal and with dense breasts is such an exemplary situation that would concur with evidence $[16,17,18]$. Also, in the private sector where availability leads to routine offering of combined DBT and 2D mammography for screening purposes, the universal risk vs. benefit principle needs to be considered. The burden of cost, over diagnosis and additional radiation to healthy women needs to be weighed against the proven increased cancer detection rates from these combined modalities when determining the screening age group and the screening interval.

Formulation and regular updating of evidence based guidelines acceptable to Sri Lanka is a current local need. Such guidelines need to be discussed in a bigger forum and should be accepted within the government and private health sectors for breast screening. Professional bodies concerning relevant disciplines such as surgery, oncology, radiology, pathology, public and community health sectors, and health administrators have a responsibility to initiate such discussion.

Does Sri Lanka need a National Breast Cancer Screening Programme?

The suitability of nationwide breast screening programmes for low and middle income countries has currently become a controversy. Cost effectiveness is considered a major determinant in this regard where cost of a national breast cancer programme is compared against life years saved by the screening programme. The IARC working group viewpoint on breast cancer screening concludes that there is currently limited evidence to confirm that mammographic breast screening can be cost effective in these middle and low income countries. It is important to highlight that the report does not mention if there is adequate data to conclude that mammographic breast screening is not cost effective for this group of countries.

India as the largest developing country in the region is offering opportunistic breast screening for women using 
both stationary and mobile breast screening facilities whilst engaging in ongoing discussions on the need and the cost effectiveness of a national screening programme $[20,21]$.

According to data from the national cancer registry of Sri Lanka, in 2007, breast cancer was the leading type of cancer in Sri Lanka with a life time risk of $2.056 \%$ for Sri Lankan women [22]. Cancer incidence data in 2009, states that the life time risk for women to develop breast cancer is $2.480 \%$, indicating that 1 in every 40 Sri Lankan women has a risk of developing breast cancer [23]. The highest incidence is seen in the 55 - 59 year age group [23].

Cancer is identified as one of the four priority noncommunicable diseases (NCD) to be prevented and controlled by 2020 by the World Health Organization (WHO) both globally and regionally [24]. In concurrence with the WHO directives, the Ministry of Health in Sri Lanka has proposed increasing the capacity for mammography in the national health sector in 2016 under the national multi-sectorial action plan for prevention and control of NCDs [25], but the need for a national breast cancer screening programme has not been addressed.

The need for a national breast cancer screening programme is a topic to be revisited in light of the current evidence and national requirement. Professional colleges, organized as social/academic bodies, concerned with relevant disciplines such as surgery, oncology, radiology and pathology hold a responsibility towards the community to make an initiative to discuss the need for a national breast cancer control programme with policy makers in the government.

The author discloses no conflict of interest. The study was conducted in accordance with the ethical standards of the relevant institutional or national ethics committee and the Helsinki Declaration of 1975, as revised in 2000.

\section{References}

1. Gilbert FJ, Tucker L, Gillan MG,, et al. Accuracy of Digital Breast Tomosynthesis for Depicting Breast Cancer Subgroups in a UK Retrospective Reading Study (TOMMY Trial). Radiology 2015 15; 142566.

2. Mandelson MT, Oestreicher N, Porter PL, et al. Breast Density as a Predictor of Mammographic Detection: Comparison of Interval- and Screen-Detected Cancers. $J$ Natl Cancer Inst 2000;92(13):1081-7.

3. Duncan KA, Needham G, Gilbert FJ, Deans HE. Incident round cancers: what lessons can we learn. Clin Radiol 1998; 53(1):29-32.

4. Carney PA, Miglioretti DL, Yankaskas BC, et al. Individual and Combined Effects of Age, Breast Density, and Hormone Replacement Therapy Use on the Accuracy of Screening Mammography. Ann Intern Med 2003 ;138(3):168-75

5. Secretan BL, Ph D, Scoccianti C, et al. Spe ci a $1 \mathrm{R}$ e $\mathrm{p}$ or $\mathrm{t}$ Breast-Cancer Screening - Viewpoint of the IARC Working Group 2014;1-6.

6. Coldman A, Phillips N, Wilson C, Decker K, Chiarelli AM, Brisson $\mathrm{J}$, et al. Pan-Canadian study of mammography screening and mortality from breast cancer. $J$ Natl Cancer Inst 2014;106(11).

7. Nelson HD, Tyne K, Naik A, Bougatsos C, et al. Screening for Breast Cancer: Systematic Evidence Review Update for the US Preventive Services Task Force: Agency for Healthcare Research and Quality (US) 2009:(Evidence Syntheses, No. 74).

8. Løberg M, Lousdal ML, Bretthauer M, Kalager M. Benefits and harms of mammography screening. Breast Cancer Res 2015;17(1):63.

9. Nelson HD, Tyne K, Naik A, Bougatsos C, et al. Screening for breast cancer: an update for the U.S. Preventive Services Task Force. Ann Intern Med 2009;151(10):727-37

10. Van Dijck JA, Broeders MJ, Verbeek AL. Mammographic screening in older women. Is it worthwhile? Drugs Aging 1997;10(2):69-79.

11. Duffy SW, Tabar L, Olsen AH, Vitak B, at al. Absolute numbers of lives saved and overdiagnosis in breast cancer screening, from a randomized trial and from the Breast Screening Programme in England. $J$ Med Screen 2010;17(1):25-30.

12. Drukteinis JS, Mooney BP, Flowers CI, Gatenby RA. Beyond mammography: new frontiers in breast cancer screening. Am J Med 2013;126(6):472-9.

13. U.S. Preventive Services Task Force website. Screening for breast cancer: recommendation statement. Published November 2009. Updated December 2009. Cited October 2015.

14. Arleo EK, Dashevsky BZ, Reichman M, Babagbemi K, Drotman M, Rosenblatt R. Screening mammography for women in their 40s: a retrospective study of the potential impact of the U.S. Preventive Service Task Force's 2009 breast cancer screening recommendations. AJR Am J Roentgenol 2013;201(6):1401-6.

15. Pisano ED, Gatsonis C, Hendrick E, et al. Diagnostic performance of digital versus film mammography for breast-cancer screening. $N$ Engl J Med 2005;353(17):1773-83.

16. Pisano ED, Hendrick RE, Yaffe MJ, Baum JK, Acharyya $\mathrm{S}$, Cormack JB, et al. Diagnostic accuracy of digital versus film mammography: exploratory analysis of selected population subgroups in DMIST. Radiology 2008;246(2):376-83.

17. Tosteson ANA, Stout NK, Fryback DG, Acharyya S, Herman BA, Hannah LG, et al. Cost-effectiveness of digital mammography breast cancer screening. Ann Intern Med 2008;148(1):1-10. 
18. Faridah Y. Digital versus screen film mammography: A clinical comparison. Biomedical Imaging and Intervention Journal 2008; 4(4).e31. Epub 2008 Oct 1

19. National Cancer Control Programme. Ministry of Health Sri Lanka. Early Detection and Management of Breast Symptoms: National Guidline for Primary Care Doctors \& Family Physicians. 2014

20. Okonkwo QL, Draisma G, der Kinderen A, Brown ML, et alde. Breast cancer screening policies in developing countries: a cost-effectiveness analysis for India. J Natl Cancer Inst $2008 ; 100(18): 1290-300$.

21. Dinshaw KA, Shastri SS, Patil SS. Cancer control programme in India: challenges for the new millennium.
Health Administrator Vol: XVII, Number 1: 10-13

22. National cancer registry, Sri Lanka- 2007, National Cancer Control Programme. Ministry of Health Sri Lanka.

23. Cancer incidence data 2009. National Cancer Control Programme. Ministry of Health Sri Lanka.

24. WHO |Global Action Plan for the Prevention and Control of NCDs 2013-2020. World Health Organization; [cited 2015 Oct 25]

25. Ministry of Health Sri Lanka. National Multisectoral Action Plan for Prevention and Control of Non Communicable Diseases 2016-1020. 